\title{
Blurring the Boundaries between Actuator and Structure: Investigating the use of Stereolithography to build Adaptive Robots.
}

\author{
Siavash Haroun Mahdavi \\ Department of Computer Science \\ University College London \\ Gower St. London WC1E 6BT, UK \\ mahdavi@cs.ucl.ac.uk
}

\author{
Sean Hanna \\ Bartlett School of Architecture \\ University College London \\ Gower St. London WC1E 6BT, UK \\ s.hanna@cs.ucl.ac.uk
}

\begin{abstract}
The aim of this work is to investigate whether stereolithographic models, combined with shape memory alloys, can be used to create robotic structures that act both as support structure and actuator. In order to test this, a structural topology was evolved and subsequently, through a deterministic process, optimised to withstand predetermined forces similar to those possibly encountered in a robot. The results show that by using this two stage process, structures can be designed and built that satisfy these requirements.
\end{abstract}

\section{Introduction}

In nature, species have evolved to best suit their niches. Monkeys have evolved to have long arms and dextrous hands that enable them to climb trees and pick fruit. Lions, on the other hand, have evolved to be strong and fast so as to most effectively catch prey in the open savannah. Each species has therefore evolved in order to best cope with typical tasks that are encountered. This can be thought of as optimising to a narrow range of tasks.

Human beings have the ability to live almost anywhere. Even early man was found in nearly all climates and surroundings. He is not the strongest, nor fastest, and he is not especially good at climbing trees either. What he has is the ability to think. Moreover, the ability to use tools to transform his body into what is needed for the environment. Weapons for hunting allow him to exceed the lion's power, and clothing can keep him warm in otherwise inhospitable climates. Therefore he has a very wide range of skills, though none are of great magnitude.

When designing a robot, it is the task of the designer to optimise the robot for the tasks it is likely to encounter, thus maximising the likelihood of success. This however is only good when you are sure of what the robot is going to encounter and the nature of its environment. In the movie 'Terminator', the robot played by Schwarzenegger was both strong and tough, thus optimised to kill! In 'Terminator 2' however, the new robot on the scene was not as strong but instead had the ability to transform its shape to best suit its environment and thus escape from unpredicted circumstances.

The ability of current robots to modify their behaviour is currently limited by their predetermined structure. In this paper, a robot structure is proposed that has not been optimised to any one task, but that can potentially fulfil a whole range of tasks. Previous work done on this type of robot has shown that such robots demonstrate the ability to adapt very closely to their environment and even exhibit the ability to recover from damage [4][5]. It is hypothesized that a more flexible structure allows the robot to better adapt in two ways:

- Increasing degrees of freedom.

- Spreading the risk of failure when the robot is damaged or situated in new environments, by allowing each part of the structure to function in different ways.

\subsection{Simple Example: A Robot Arm}

In order to illustrate this idea, a robot arm designed in the standard way is compared to one created by the method proposed in this paper. The support structure for the standard arm is rigid, with a hinge in the middle. The actuator is separate and upon compression, induces a rotating motion about the hinge. See Fig. 1.
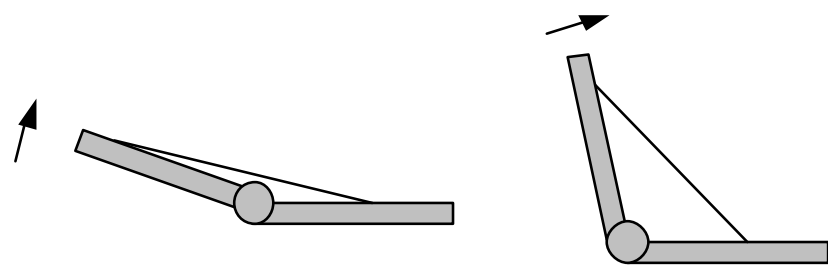

Fig 1. A standard robot arm rotating about the hinge.

This produces a limited range of motion. However, by constructing the robot arm from a structure with varying degrees of density and flexibility, a robot arm can be created that has many advantages. By spreading the actuation throughout the structure, many more potential degrees of freedom can be satisfied. This enables the arm to have the ability to move in ways not previously determined by the designer, see Fig. 2 .
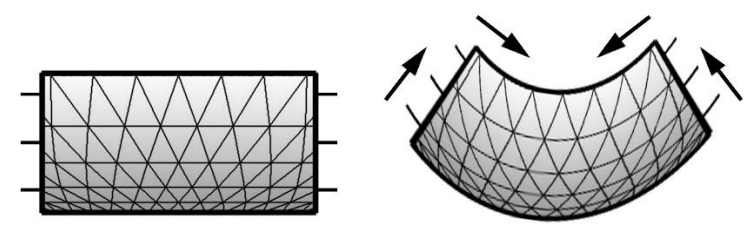

Fig 2. Flexible structure with distributed actuation. 
As this example illustrates, this proposed method has significant benefits and applications in respect to its adaptability. We propose constructing the robot using a technique known as stereolithography, combined with shape memory alloys (see background sections 2.1 and 2.2 for details.) Therefore this work is an initial investigation to see whether the stereolithographic structures can be designed that can be adapted to varying force and flexibility requirements. This work will then lead the way to optimising these structures to real forces that are present within real robots using finite element analysis.

\section{Background}

\subsection{Stereolithography: The Process}

Stereolithography is a method of creating physical 3D realisations of CAD models; see [2] for a fuller explanation. It is one of the many types of machines collectively called 'rapid prototyping machines'. As the name suggests, their primary usage is with the rapid building of prototypes for testing by engineers and designers. However as the technology has been dramatically improving over the past several years, it has become evident that this process can be used for more than building prototypes and can be itself a method for constructing parts.

The stereolithography machine consists of a tank filled with liquid photopolymer which is sensitive to ultraviolet light. An ultraviolet laser 'paints' one of the layers, exposing the liquid in the tank and hardening it, a platform then drops down into the tank a fraction of a millimetre and the laser paints the next layer. This process repeats until the model is complete.

Once completed, the object is rinsed with a solvent and then baked in an ultraviolet oven that thoroughly cures the plastic.

\subsection{Shape Memory Alloys}

Nitinol, an alloy made of Nickel and Titanium, was developed by the Naval Ordinance Laboratory. When current runs through it, thus heating it to its activation temperature, it changes shape to the shape that it has been 'trained' to remember. The wires used in this project simply reduce in length, (conserving their volume and thus getting thicker), by about 5-8 \% [8].

Shape Memory Alloys (SMAs), when cooled from the stronger, high temperature form (Austenite), undergo a phase transformation in their crystal structure to the weaker, low temperature form (Martensite). This phase transformation allows these alloys to be super elastic and have shape memory [8].

The phase transformation occurs over a narrow range of temperatures, although the beginning and end of the transformation actually spread over a much larger range of temperatures. Hysteresis occurs, as the temperature curves do not overlap during heating and cooling [8]. With thick wires, this could bring about problems for a robot as the NiTi wires would take some time before returning to their original lengths, however, due to the very small diameter of the NiTi wires used $(\sim 0.15 \mathrm{~mm})$, the hysteresis is almost negligible as the cooling to below the Martensite temperature, (Mf), is almost instantaneous [3]. See Fig. 3.

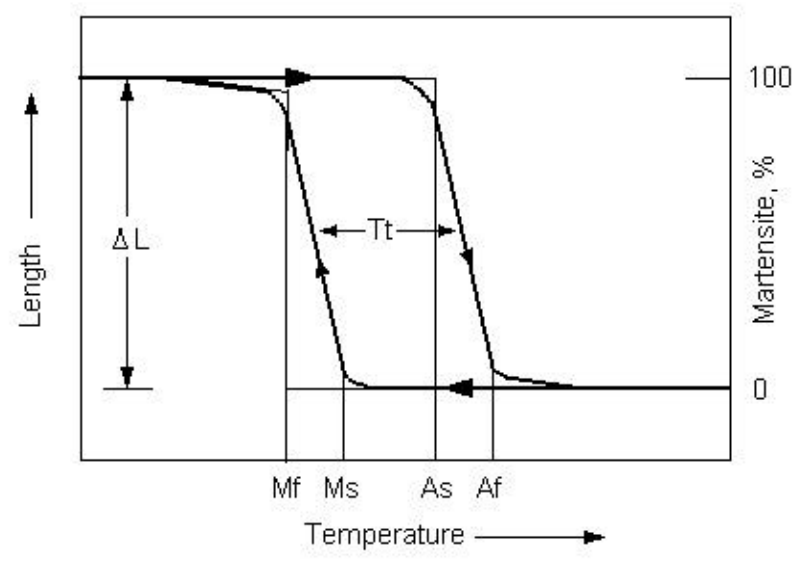

Fig. 3. Graph showing change in length during heating and cooling. The hysteresis is represented by Tt.

\subsection{Robots that Use Rapid Prototyping}

Most work that has been done using rapid prototyping technologies has been in just that: the use of these technologies to rapidly produce prototypes. In general these are designed to be manufactured by other means.

There are however some researchers that have attempted to use rapid prototyping as a means to manufacture robots. The most prominent project is the Golem Project where a robot topology was evolved in a simulator and the body was then manufactured using a form of rapid prototyping called fused deposit modelling (FDM) [9]. There is no cost associated with complexity and so very detailed structures can be constructed. This level of detail and resolution has been exploited by several teams that have used rapid prototyping techniques (stereolithography) to make very small robots [11][7]. Similar work to that described in this paper has been done by NASA, investigating whether it is possible to use rapid prototyping (stereolithography) and SMAs together to create a very adaptive robot. Their robot resembles a bush and so has been aptly called a 'bush robot' [10].

\subsection{Self Adapting Snake Robots}

Previous work by one of the authors involved a robot snake that was constructed entirely out of wood, foam and NiTi, (excluding the circuit board and wiring of course)[4]. The controller for the robot snake's motion was an evolved Finite State Machine. The type of learning was online and so the snake was constantly learning how to move. This meant that it could adapt to changing environments. Indeed, an experiment was done where the NiTi wires were deliberately damaged to see whether the robot snake could evolve a new method of locomotion by using its remaining NiTi wires to best recover its motion [5]. 
The main bulk of the robot snake is made of foam. This provides a restoring force great enough to restore the NiTi wires to their original lengths after each activation. The robot snake used in the experiments uses twelve NiTi wires (diameter $=0.176 \mathrm{~mm}$, activation (Austenite) temperature $=70^{\circ} \mathrm{C}$, recommended current $200 \mathrm{~mA}$ ). The body of the robot snake was split into four segments (this is an extension from the first robot snake prototype described in [4] which used only a single segment). These segments are readily detachable from each other and the whole structure could be expanded or segments replaced. Each segment had three NiTi wires running down its length and a central copper wire that ran through the foam and supplied the power, much like a spinal chord carries nerve impulses to muscles through the body see Fig. 4. These four segments were connected together and the ends of the 'spinal chord' were joined to create a continuous connection along the length of the robot snake, see Fig. 5. The total weight of the robot snake was approximately $150 \mathrm{~g}$.

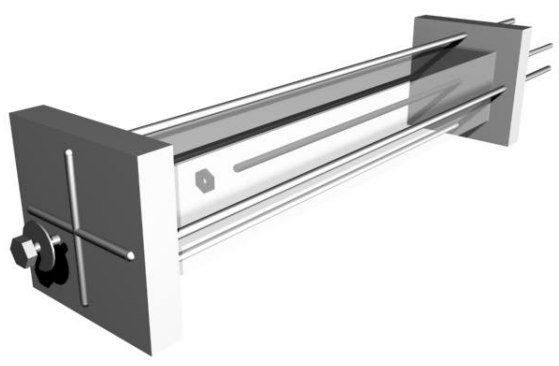

Fig. 4 One segment of the Self Adapting Snake

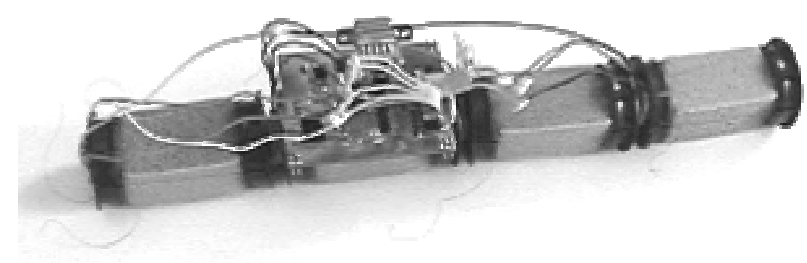

Fig. 5 A photo of the Self Adapting Snake

The method in this paper would replace the wood and foam structure with one continuous form that is optimised at each point to what it needs to do. Therefore, in the snake robot example, alternating layers of wood and foam would be replaced by a single stereolithographic structure that was rigid at some points and flexible at others.

By gradually morphing between the rigid and flexible parts of the robot, the boundaries between these normally separate components have been blurred. This minimises the effect of damage, for example, as no one part of the structure is critical (i.e. spreading risk).

\subsection{Stereolithography and Structural Opti misation}

Evolutionary algorithms are an increasingly popular technique of design and optimisation that operate on genetic principles; see [1] for an introduction. Previous work by the authors involved the utilization of an evolutionary algorithm to evolve the microstructure of an object created by a stereolithography machine [6].

This structure was optimised to withstand loads applied to it while at the same time minimizing overall weight. A two part algorithm was proposed that evolved the topology of the structure with a genetic algorithm, while calculating the details of the shape with a separate, deterministic, iterative process derived from standard principles of structural engineering. The division of the method into two separate processes allowed both flexibility to changed design parameters without the need for re-evolution, and scalability of the microstructure to manufacture objects of increasing size.

Standard vector methods were used in the analysis of the structures. Given a tensile or compressive force $\mathbf{F}$ acting in the direction of the structural element this can be decomposed into its $(\mathrm{x}, \mathrm{y}, \mathrm{z})$ components along each of the axes. For each node point to be in a state of equilibrium, all of its connecting members are considered using the element analysis equations, such that:

$$
\mathrm{S}_{\mathbf{F}_{\mathrm{x}}}=0 \quad \mathrm{~S} \mathrm{~F}_{\mathrm{y}}=0 \quad \mathrm{~S} \mathrm{~F}_{\mathrm{z}}=0
$$

The simultaneous analysis of multiple elements is performed using the direct stiffness method (DSM).

Ten thousand generations of the Genetic Algorithm under equilibrium loading conditions resulted in the structure shown in Fig. 6. Results showed that a structure was evolved that was both light and stable. The overall shape of the evolved lattice resembled a honeycomb structure that also satisfied the restrictions imposed by the stereolithography machine.

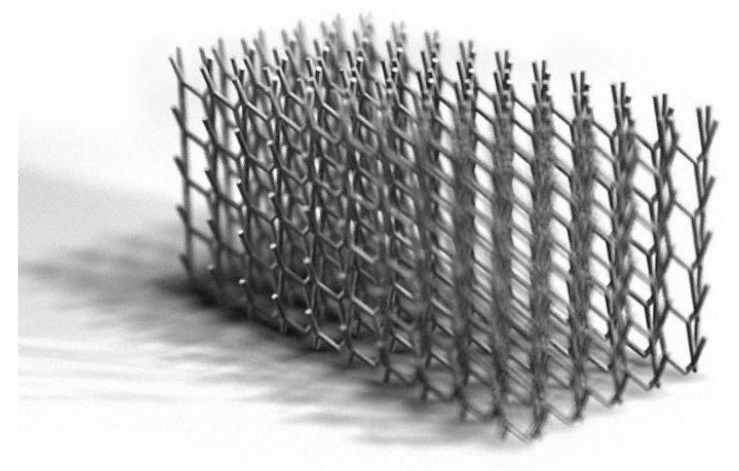

Fig. 6. The stereolithographic model created by the genetic algorithm. 


\section{Method}

\subsection{Overview: Robot}

We use the topology of the honeycomb structure above, which has been optimised for equilibrium loading conditions. As a possible structure for a robot, the shape of this is modified at each point to yield varying degrees of resistance and flexibility. $\mathrm{NiTi}$ wires would run through the structure, as through the foam in the robots in sec. 2.4 , inducing movement by contorting it into different shapes.

\subsection{The Structure}

By modifying the internal structure of the 'foam' in different regions, the robot, via NiTi wire actuation, will be able to achieve many desired configurations. The goal then is to produce a structure that resists a force of a different direction and magnitude at each point (i.e. it may be more flexible to accommodate a horizontal NiTi wire in one region, and more flexible to a vertical in another) but does not have any discontinuities to weaken it.

The structure of the robot material consists of a three-dimensional lattice of struts oriented to optimise its efficiency for the stresses incurred at that particular point. It is described by a series of connected node points in space, in which the connections between them (the topology) describe the struts and the positions of the node points describe the shape. By separating the topology of strut connections from the actual shape and position of those struts, a two-stage algorithm is used to determine the form of the structure. As described above (sec. 2.5) The topology is evolved by a genetic algorithm (GA), and the node positions are set by a deterministic analysis of the forces by the direct stiffness method (DSM).

The overall volume of the robot shape is divided into a grid of equal unit cubes, each of which is to contain a topologically identical structure but is to have differing structural properties in terms of the magnitude or direction of force to be resisted. The structure of each cube is connected to each of its neighbours to form a continuous structure. (See [6] for more on evolving the topology.)

Given a single suitable strut topology, it is therefore possible to modify the shape so that the structure reaches a state of equilibrium for each applied force $\mathbf{F}$. One unit cube is analysed by the DSM, which yields the stresses of each member, and each node point is then moved to the point that brings its local forces into a state of equilibrium. After a number of iterations the shape converges to its final state.

The shape can change in two ways: the node points defining the connections between the struts can move in space, and the thickness of each strut can itself change. Both of these are determined by the DSM. Applying the iterated analysis for different applied force vectors finds different equilibrium conditions, thereby changing the lengths and relative angles between struts, and the thickness of their cross sections.

Modification to the structure is performed by this second part of the algorithm: by applying a simulated force to a specific unit cube, the iterated DSM algorithm finds the shape best suited to resist it. By varying the force slightly, one cube becomes slightly different from its neighbour, thus producing a smooth continuous transition from one condition to the next. The same topology is used throughout, as breakpoints would represent structural discontinuity and be structural failures otherwise.

A simulated force is specified as a three-dimensional vector, in which a positive or negative magnitude indicates a tensile or compressive force in each of the $x$, $y$ and $z$ axes. Specific forces are assigned to several unit cubes within the structure (the zones designated for NiTi wires) and every other unit cube is then assigned a linear combination of these force vectors. This results in a three-dimensional grid of force vectors in which each varies slightly from its immediate neighbours.

The final structure is then built up by applying the iterative DSM method to the strut topology using each of the force vectors to generate node positions for each unit cube. Struts are placed between the nodes within the cube according to the topology, and connecting the slightly differing positions of the nodes in each neighbouring cube. The final iteration of the DSM analysis provides the tensile or compressive force along the axis of each strut given the applied force vectors. The magnitude of this force is multiplied by a normalization constant (no real-world units are used) and used as the cross-sectional area of each strut.

\section{Results and Analysis}

\subsection{Force Conditions}

Two different force conditions were applied to the structurally stable strut topology shown in Fig. 8. For clarity, one condition involved varying only the magnitude of the forces, and the other only the direction. Structures A and B (see Fig. 7) are each composed of a grid of 5 by 5 by 15 unit cubes, and a continuously varied force vector from the bottom to the top of the structure.

Structure A varies only the magnitude, with a force vector of equal tension in all three axes that decreases from $[50,50,50]$ at the bottom to $[1,1,1]$ at the top. Structure B maintains the same overall magnitude, but changes the direction of the vector from $[50,1,1]$ (the $\mathrm{x}$-axis) at the bottom to $[1,50,1]$ (the $\mathrm{y}$-axis) at the top. 

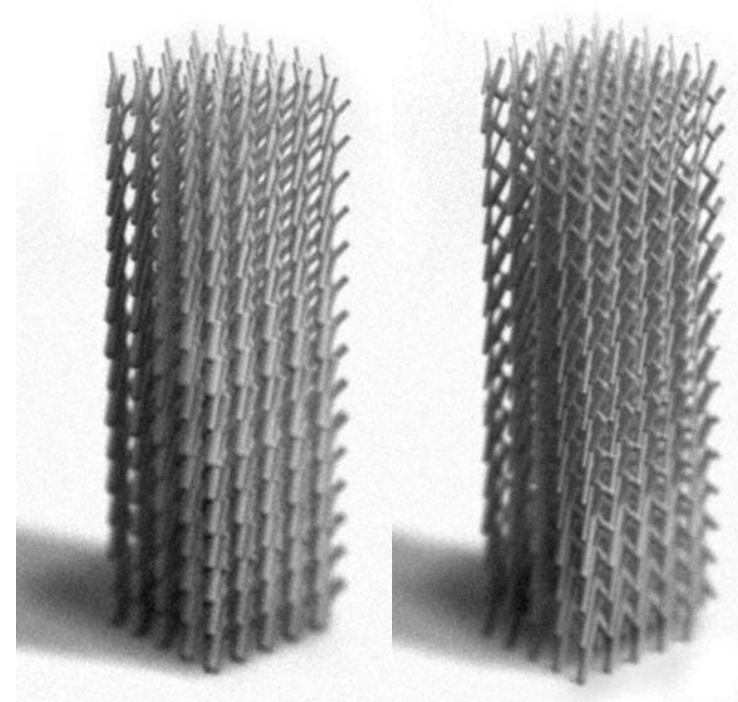

Fig 7. Structure A (left) and Structure B (right)

\subsection{Observations: Structure A}

The side profile of Structure A reveals a gradual decrease in thickness of the struts from the bottom to the top of the structure in accordance with the gradual decrease in magnitude of the force vector. (See Fig 8 a) The extremes can be examined in greater detail in the top views of the structure taken from the top and bottom of the structure. (See Fig 8 b\&c) Two observations may be noted in these details. First, because the force vector is equal in all three axes each strut is the same width within the unit cube. The second observation is that because the force vector doesn't change in orientation, the shape of the strut configuration as given by the node point positions and strut lengths does not change.

\subsection{Observations: Structure B}

Structure B is the result of varying the direction of the applied force, and again shows a continuous change in geometry in the side profile (Fig. 9 a), but in this case the change is more complex. The top views of the bottom and top layers of the structure (Fig 9 b\&c) correspond to applied force vectors in the directions of the $\mathrm{x}$ and $\mathrm{y}$-axes respectively, and in these images the shape change is obvious. At the bottom the force is oriented in the direction of the $\mathrm{x}$-axis (the horizontal in the bottom image) and all struts except one in each unit are approximately aligned to this direction in the $x-y$ plane. At the top the applied force is oriented along the y-axis (vertical in the top image), and all struts except one are aligned toward this direction. Moreover, the struts in the direction of the y-axis are markedly thicker in cross section than the single strut running roughly perpendicular to the force vector. Structure B has compensated to resis the force vector in two ways: the node points have moved to orient the struts in the direction of the force vector, and the width of the struts has varied within each unit cube to respond to increased force in that direction.
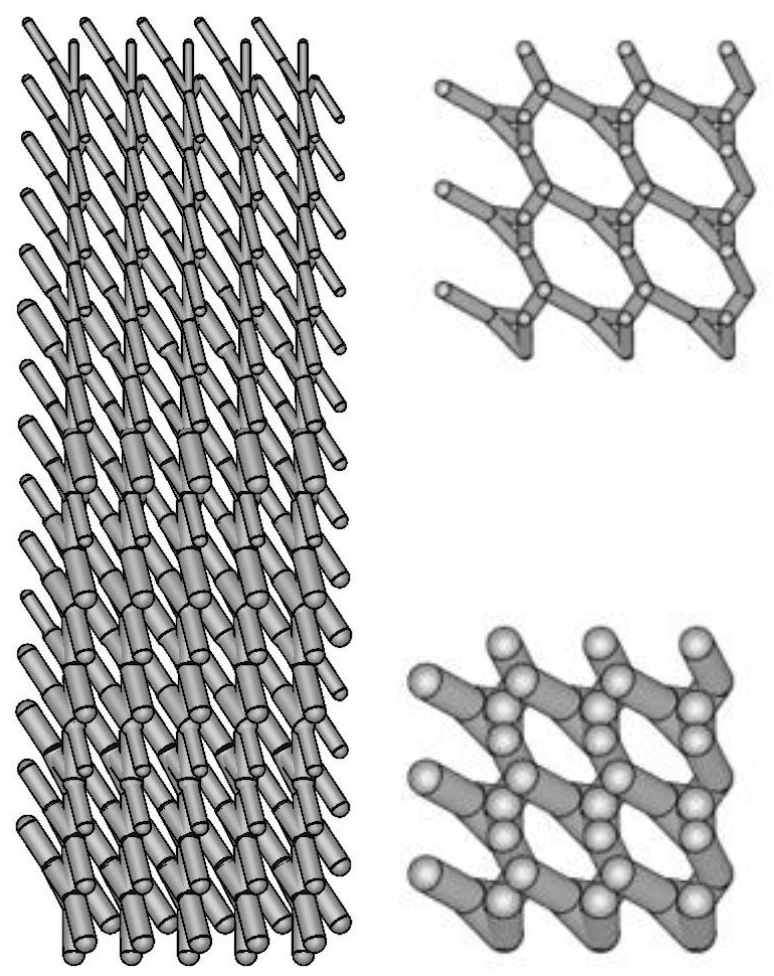

Fig 8. Structure A: Side view (a), and slices from the top (b) and bottom (c)
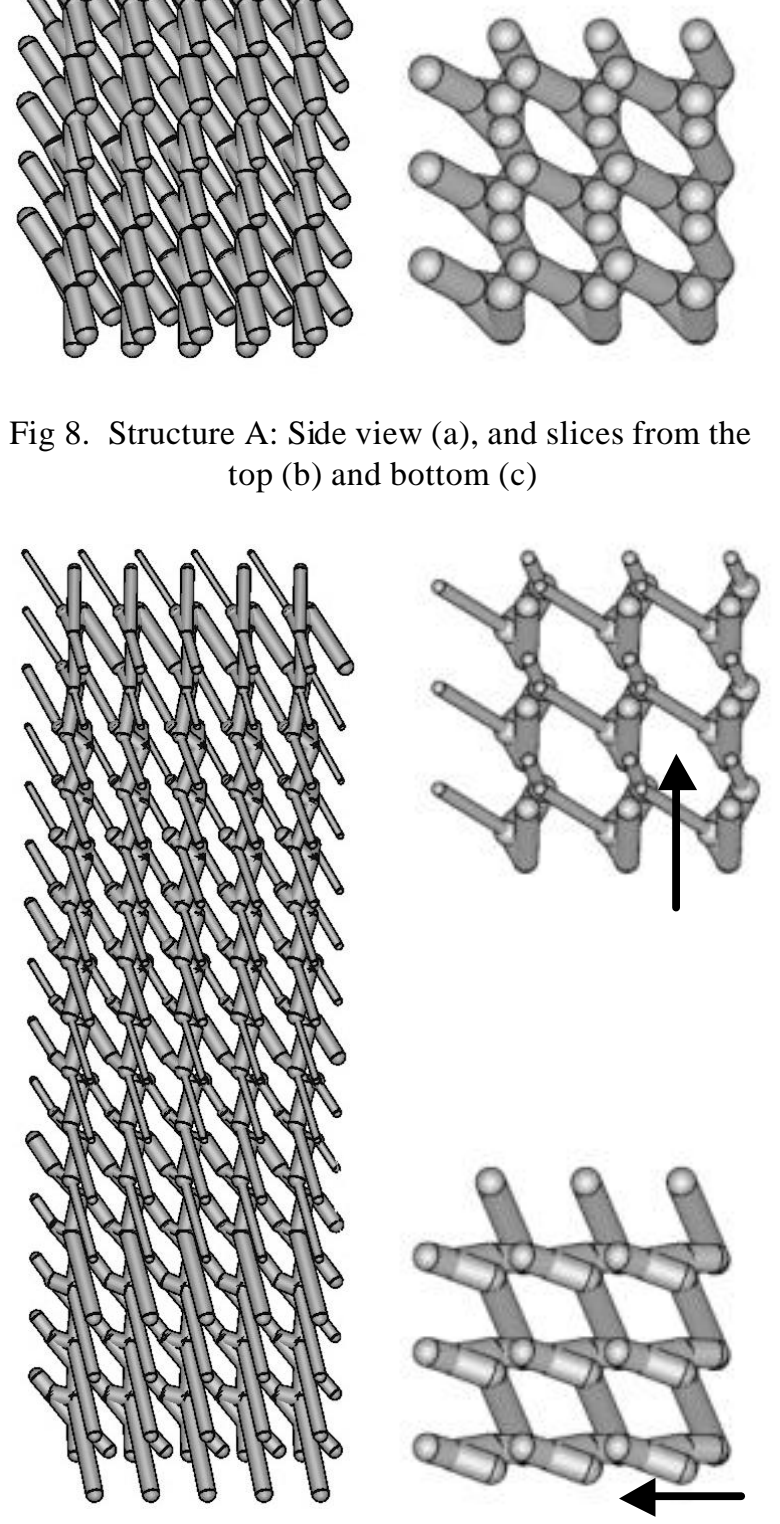

Fig 9. Structure B: Side view (a), and slices from the top (b) and bottom (c) 
Empirical tests confirmed that the two structures (A and B) behave as predicted above. Because the structures were optimised to forces that were unitless, the testing that was performed took the form of applying the same force along the length of the structure and observing by how much the structure depressed at various points.

\section{Conclusion}

A method has been investigated for creating structures that could be optimised to counteract forces similar to those present in a proposed robot. A structure with an evolved topology was taken and, using deterministic methods, constructed to deform under distributed actuation. Analysis of the two examples allows several conclusions to be drawn on the performance of the method used:

- Continuous incremental change in the forces applied to the unit cubes results in a structure that blends smoothly between geometrical states.

- Changes in the magnitude of the force vector cause corresponding proportional changes in the diameter of the struts.

- Changes in the direction of the force vector cause corresponding changes in the orientation and length of the struts.

- The force vector determines the stiffness of the resulting structure, or its resistance to compression in any direction.

\section{Future Work}

This paper investigated whether it would be possible for stereolithographic models to morph to counteract predetermined forces with the intention of extending it to real robot applications. The next step in this work would be to design a robot and by using finite element analysis, determine where, in what direction and at what magnitude, forces were present within an actual robot design. The method used here would then take in these parameters and generate a structure that is best suited for the job. Future work will investigate how a genetic algorithm or other learning algorithms allow these robots to move and adapt in their environments.

\section{References}

[1] Bentley, P. J. (Ed.) (1999) EvolutionaryDesign by Computers. Morgan Kaufmann Pub.

[2] Brain, Marshall.(2002) "How Stereolithography Works," www.howstuffworks.com/stereolith.htm.

[3] Haroun Mahdavi, S. (2002) Evolving Motion Master's Dissertation, MSc IS, University College London, Dept. Computer Science.

[4] Haroun Mahdavi, S. and Bentley, P. J.(2003) Evolving Motion of Robots with Muscles. In Proc. of EvoROB2003, the 2nd European Workshop on Evolutionary Robotics, EuroGP 2003. pp. 655-664.

[5] Haroun Mahdavi, S. and Bentley, P. J. (2003) An Evolutionary Approach to Damage Recovery of Robot Motion with Muscles. Proc. of the European Conference on Artificial Life (ECAL 2003).

[6] Haroun Mahdavi, S and Hanna, S. (2003) "An Evolutionary approach to microstructure optimisation of stereolithographic models." Proceedings of CEC203. The Congress on Evolutionary Computation.

[7] Heller, E and Adkin, D (2001), "Mini Robot Research",http://www.sandia.gov/media/NewsRel/NR2001 /minirobot.htm

[8] Hodgson, Darel E. (2002), Shape Memory Applications, Inc., Ming $\mathrm{H}$. Wu, Memory Technologies, and Robert $\mathrm{J}$. Biermann, Harrison Alloys, Inc. www.sma.com/Shape Memory Alloys.htm

[9] Lipson, H and Pollack, J.B (2000), "Automatic design and Manufacture of Robotic Lifeforms", Nature 406, pp. 974978.

[10] Moravec, H and Easudes, J (1999), Final Report NASA ACRP NCC7-7, "Bush Robots".

[11] Reshko, G et al. (2000), "Rapid Prototyping for small robots", www-2.cs.cmu.edu/ reshko/Publications/ prototy ping.pdf 